\title{
A Bibliographic Database Search Strategy Informed by an Operational Definition of Complementary, Alternative, and Integrative Medicine
}

Jeremy Y. Ng ( $\nabla$ ngjy2@mcmaster.ca )

McMaster University

Tushar Dhawan

McMaster University

Ekaterina Dogadova

McMaster University

Zhala Taghi-Zada

McMaster University

Alexandra Vacca

McMaster University

Renee-Gabrielle Fajardo

McMaster University

Hooriya A. Masood

McMaster University

Riva Patel

McMaster University

Samira Sunderji

McMaster University

L. Susan Wieland

University of Maryland School of Medicine

David Moher

Ottawa Hospital Research Institute

\section{Research Article}

Keywords: complementary and alternative medicine, integrative medicine, operational definition, standard of classification, bibliographic database, medical research platform, search strategy

Posted Date: March 7th, 2022

DOI: https://doi.org/10.21203/rs.3.rs-1390385/v1 
License: (c) (i) This work is licensed under a Creative Commons Attribution 4.0 International License. Read Full License 


\section{Abstract \\ Background}

Determining which therapies fall under the umbrella of complementary, alternative, and/or integrative medicine (CAIM) is difficult for several reasons. An operational definition is dynamic, and changes depending on both historical time period and geographical location, with many countries integrating or considering their traditional system(s) of medicine as conventional care. We have previously reported the first operational definition of CAIM informed by a systematic search. In the present study, we have developed a bibliographic database keyword search strategy for CAIM based on this operational definition, in order to standardize research involving systematic searches such as systematic reviews and bibliometric analyses.

\section{Methods}

Using the finalised operational definition of CAIM's 604 therapies, a single keyword search strategy was developed for the most common bibliographic databases, including those searchable on the OVID platform (e.g., MEDLINE, EMBASE, PsycINFO, AMED), the EBSCO platform (e.g., ERIC, CINAHL), Scopus, and Web of Science. The Therapeutic Research Center's "Natural Medicines" database was searched for all 604 therapies, and each item's scientific name and/or synonym was included as a phrase to the search strategy.

\section{Results}

This developed bibliographic database search strategy provides a standardised list of CAIM terms that may be searched on bibliographic databases including those found on the OVID platform (e.g., MEDLINE, EMBASE, PsycINFO, AMED), the EBSCO platform (e.g., ERIC, CINAHL), Scopus, and Web of Science.

\section{Conclusion}

Researchers can select relevant terms for their CAIM study and insert the keywords or phrases into these databases to receive all accessible data. This search technique can simply be copied and pasted into the search bar of each database to identify research by keywords, which is the most inclusive, or by words in the article title, which is more selective. Given its versatility across multiple commonly used academic platforms/databases, it is expected that this search strategy will be of great value to those conducting research on CAIM topics involving systematic searches.

\section{Introduction}


Complementary, alternative and integrative medicine (CAIM) refers to non-mainstream practices that are used together with, in place of, or in coordination with conventional medicine, respectively [1]. However, the identification of a unified set of CAIM therapies present challenges: there may be limited recognition of therapies that stand alone or exist without a complementary, alternative or integrative relationship to conventional medicine, and there is a lack of universal consensus regarding how to accurately label a given CAIM therapy, even in medical literature [2]. Despite these challenges, patients continue to use CAIM globally. For example, of the 170 countries that comprise the World Health Organization, $88 \%$ adopted policies and programs for CAIM [3, 4]. Patients may actively choose to use CAIM therapies to improve physical and mental aspects of their well-being, such as achieving symptom relief and a sense of control over their own care, respectively [5-7]. These patient preferences propel research efforts in the CAIM field, as new evidence allows healthcare practitioners to engage in shared decision-making with their patients $[2,8]$.

Since there is no standard list of CAIM therapies that is mutually agreed upon by the research community, certain research studies, including umbrella, scoping, and systematic reviews and bibliometric analyses, that aim to be comprehensive about CAIM, are methodologically limited. The failure to include a comprehensive list of CAIM therapies in a search strategy can lead to the omission of eligible research studies, which may result in a study arriving at an inaccurate conclusion. Since the 1940s, the volume of CAIM publications has followed an upward trend, with 2020 being the most productive year globally to date [46]. To continue this trend, a comprehensive operational definition, and by extension a search strategy, of CAIM is needed. So far, only one operational definition of complementary and alternative medicine (CAM) has been published by Wieland et al. (2011). However, this definition was developed for the purpose of selecting relevant systematic reviews from a single journal, did not consider the "integrative" component of CAIM, considered only two sources (the MeSH (Medical Subject Headings) definition of complementary therapies and the Complementary Medicine subset search strategy in PubMed) as potential sources of an operational definition, and was not designed to result in a search strategy. Nevertheless, Wieland et al.'s sentiment of supporting the harmonization of CAIM research with a more standardized and precise classification of CAIM therapies that would enable better collaboration among researchers, clinicians, and others who seek a comprehensive picture of CAIM is shared by the present study [9]. Thus, the objective of this study is to construct a keyword search strategy for use in common bibliographic databases based on the operational definition of CAIM we have described in a separate publication.

\section{Methods}

\section{Previous study: CAIM Operational Definition}

We have previously reported the first operational definition of CAIM informed by a systematic search, which serves as the basis for the present study [47]. Briefly, the CAIM operational definition was derived from four types of quality-assessed media types: 1) peer-reviewed articles from the MEDLINE, EMBASE, AMED, PsycINFO, CINAHL, Scopus and Web of Science databases; 2) the "Aims and Scopes" of peer- 
reviewed CAIM journals; 3) CAIM entries in highly accessed online encyclopaedias; and 4) websites resulting from Health On the Net Code of Conduct (HONcode) searches. Across these four media types, eligible items were reviewed for CAIM or CAIM-related terms and data extracted for the operational definition. The Methods section of our previous study describes our eligibility criteria, searching and screening, data extraction and analysis, and the creation of a CAIM operational definition. A protocol was registered with the Prospective Register of Systematic Reviews (PROSPERO), registration number CRD42020206301.

\section{Development of the Bibliographic Database Search Strategy}

Using the 604 therapies in the finalized operational definition of CAIM, one search strategy, using keywords, was developed for the most commonly used bibliographic databases including those searchable on the OVID platform (e.g. MEDLINE, EMBASE, PsycINFO, AMED), the EBSCO platform (e.g. ERIC, CINAHL), as well as Scopus and Web of Science. To accomplish this, all 604 therapies were searched on the Therapeutic Research Center's "Natural Medicines" database, and every relevant monograph's scientific name and/or synonym was added as a term to the search strategy. We opted to exclude controlled vocabularies from our search strategy for two main reasons: 1) this would require customized search strategies for each database, and 2) this would be impractical, given that many databases regularly update their indexing terms (for example, MEDLINE updates their MeSH terms annually: https://www.nlm.nih.gov/mesh/whatsnew.html). We also reviewed common features that are recognized across all the aforementioned platforms/databases, opting to use asterisks $\left({ }^{*}\right)$ as opposed to other wildcard symbols. The "OR" Boolean operator was used to separate each individual search term. To improve specificity, we identified and removed search terms as follows: 1) acronyms (i.e. "TENS" as an acronym for "transcutaneous electrical nerve stimulation"); 2) element symbols (i.e. "Fe" as the symbol for "iron"); and 3) amino acid 3 letter codes (i.e. "Arg" as the code for "arginine"), as it is generally not common practice to include just the acronyms, element symbols, or amino acid 3 letter codes alone in titles and abstracts. Lastly, we also identified and removed 4) other terms that had the potential to generate a disproportionate volume of non-CAIM-specific search results (i.e. "May" as a synonym for "Hawthorn" (Crataegus spp.)); these terms were identified through standalone searches conducted on MEDLINE whereby the first line of results were visually scanned for a large proportion of non-CAIMspecific search results. Before terms were excluded, we searched them on multiple databases and reviewed the first page of results to verify that non-CAIM-specific search results were being retrieved.

\section{Results}

\section{Bibliographic Database Search Strategy}

This formulated bibliographic database keyword search strategy provides a standardized list of CAIM terms that is searchable on frequently used databases including those found on the OVID platform (e.g. MEDLINE, EMBASE, PsycINFO, AMED), the EBSCO platform (e.g. ERIC, CINAHL), as well as Scopus and Web of Science. Searchers may identify the terms that are relevant to their CAIM focus and enter the 
keywords or phrases on these databases to retrieve all available data. This search strategy can simply be pasted into each database's search field to identify studies by keywords, which is most inclusive, or by words in the article title, which is more selective. The search strategy is available for download as

Supplementary File 1. When applying the search strategy to a given bibliographic database or platform it is recommended to use the respective "advanced search" feature; in most cases, it can simply be copied and pasted directly into the advanced search bar. Additionally, and for the purpose of increased transparency, a complete list of all excluded terms and their corresponding reason for removal is available for download as Supplementary File 2.

\section{Discussion And Conclusion}

In the present study, one search strategy, using keywords, was developed based on the CAIM operational definition we reported in our previous study. Prior to this, Wieland et al. (2011) developed an operational definition of CAM consisting of 70 therapies and the Cochrane Complementary Medicine website amplified this by listing 259 CAIM therapy terms [10]. Our previously reported study represents the largest operational definition of CAIM created to date, with 604 CAIM therapy terms and their respective synonyms; in addition, it is the only systematically and transparently developed operational definition of its kind [47]. The present study is the first to present a comprehensive keyword search strategy to identify CAIM studies in the literature for systematic searches.

Umbrella, scoping, and systematic reviews as well as bibliometric analyses are areas in which a CAIM search strategy would be of great utility. These are research methodologies that gather literature published in a certain academic discipline, either to fill a knowledge gap for systematic and scoping reviews, or to identify characteristics of the literature, such as the impact factor, for bibliometric analyses [11-21]. Prior to this study, different means were used by different researchers to capture CAIM-related literature for systemic and scoping reviews, which introduced selection biases into their search results [22]. Likewise, bibliometric analyses generally assessed CAIM research from CAIM journals [23-25], based on research methodologies [26, 27], or specific CAIM interventions [28-33], omitting additional studies that might have been captured with a comprehensive list of CAIM therapies. Fortunately, standardized academic search strategies allow researchers to obtain more consistent search results and consequently find a plethora of relevant peer-reviewed literature and accurately gauge the current status, as well as the future directions, of CAIM research in their respective fields [34-38]. It can be anticipated that should researchers conducting future reviews and analyses use the search strategy presented in this study, they will identify a more comprehensive collection of eligible general CAIM studies or literature characteristics.

Despite its utility, there are challenges and limitations using this search strategy to seek out general CAIM literature. There are various frameworks regarding what constitutes CAIM across different countries and cultures; thus, the search strategy may result in under- or over-inclusiveness of certain therapies as there is still no consensus, even among experts, regarding what constitutes a CAIM therapy [22, 39]. For example, CAIM therapies that originate from Buddhist practices may be considered part of conventional 
care in Eastern countries such as Thailand and Sri Lanka while European and North Americans countries may consider these practices as CAIM [40,41]. Similarly, there is also an English language bias, as the search strategy was derived from the operational definition which was constructed based on English literature only. The majority of CAIM studies are published in the English or Chinese languages and interestingly, English journals have been found to deviate towards reporting the negative results of CAIM use, while Chinese journals report positive results [27, 42, 43]. As a result, the language of publication may influence which therapies are discussed by a study, and consequently which were included in our list $[44,45]$. Lastly, a limitation inherent to search strategies in general also exists but is augmented by the dynamic nature of the CAIM field. There is a trade-off between recall and precision in terms of results retrieval. If a search term is too ambiguous or broad, there will likely be 'noise' that researchers will have to sift through [38]. Alternatively, if a search term is too specific or narrow, eligible articles may be missed. While we have prioritized optimizing both recall and precision, the differences in CAIM therapy description by country, culture, language, system of traditional medicine and school of thought looms, nevertheless.

\section{List Of Abbreviations}

CAIM: complementary, alternative and integrative medicine

CAM: complementary and alternative medicine

HONcode: Health On the Net Code of Conduct

PROSPERO: Prospective Register of Systematic Reviews

\section{Declarations}

\section{Ethics Approval and Consent to Participate}

Not applicable.

\section{Consent for Publication}

All authors consent to this manuscript's publication.

\section{Availability of Data and Materials}

All relevant data are included in this manuscript.

\section{Competing Interests}

The authors declare that they have no competing interests.

\section{Funding}


This study was unfunded.

\section{Authors' Contributions}

JYN: designed and conceptualized the study, collected and analysed data, drafted the manuscript, and gave final approval of the version to be published.

TD: collected and analysed data, critically revised the manuscript, and gave final approval of the version to be published.

ED: collected and analysed data, critically revised the manuscript, and gave final approval of the version to be published.

ZT: collected and analysed data, critically revised the manuscript, and gave final approval of the version to be published.

AV: collected and analysed data, critically revised the manuscript, and gave final approval of the version to be published.

R-GF: collected and analysed data, critically revised the manuscript, and gave final approval of the version to be published.

HAM: collected and analysed data, critically revised the manuscript, and gave final approval of the version to be published.

RP: collected and analysed data, critically revised the manuscript, and gave final approval of the version to be published.

SS: collected and analysed data, critically revised the manuscript, and gave final approval of the version to be published.

LSW: assisted with the analysis of data, critically revised the manuscript, and gave final approval of the version to be published.

DM: assisted with the analysis of data, critically revised the manuscript, and gave final approval of the version to be published.

\section{Acknowledgements}

JYN was awarded a Research Scholarship and an Entrance Scholarship from the Department of Health Research Methods, Evidence and Impact, Faculty of Health Sciences at McMaster University.

\section{References}


1. National Center for Complementary and Integrative Health $(\mathrm{NCClH})$. Complementary, Alternative, or Integrative Health: What's In a Name? https://www.nccih.nih.gov/health/complementary-alternativeor-integrative-health-whats-in-a-name Accessed February 09, 2022.

2. Ng JY, Boon HS, Thompson AK, Whitehead CR. Making sense of "alternative", "complementary", "unconventional" and "integrative" medicine: exploring the terms and meanings through a textual analysis. BMC Complement Altern Med. 2016; 16:134. https://doi.org/10.1186/s12906-016-1111-3

3. World Health Organization. Traditional, Complementary and Integrative Medicine. 2021. https://www.who.int/health-topics/traditional-complementary-and-integrative-medicine\#tab=tab_1 Accessed February 09, 2022.

4. World Health Organization. WHO global report on traditional and complementary medicine 2019. 2019. https://apps.who.int/iris/handle/10665/312342 Accessed February 09, 2022.

5. Li J, Zhu J, Hu H, Harnett JE, Lei Cl, Chau KY, Chan G, Ung COL. Internationalization of Traditional/Complementary Medicine products: market entry as medicine. Chinese Medicine. 2018; 13(1):50. https://doi.org/10.1186/s13020-018-0209-6

6. Chao J, Dai Y, Verpoorte R, Lam W, Cheng YC, Pao LH, Zhang W, Chen S. Major achievements of evidence-based traditional Chinese medicine in treating major diseases. Biochem Pharmacol. 2017; 139:94-104. https://doi.org/10.1016/j.bcp.2017.06.123

7. Astin JA. Why patients use alternative medicine: results of a national study. JAMA. 1998; 279(19):1548-1553. https://doi.org/10.1001/jama.279.19.1548

8. Redvers N, Marianayagam J, Blondin B. Improving access to Indigenous medicine for patients in hospital-based settings: a challenge for health systems in northern Canada. Int J Circumpolar Health. 2019; 78(2):1589208. https://doi.org/10.1080/22423982.2019.1589208

9. Chopra A, Saluja M, Tillu G, Sarmukkaddam S, Venugopalan A, Narsimulu G, Handa R, Sumantran V, Raut $A$, Bichile $L$ et al. Ayurvedic medicine offers a good alternative to glucosamine and celecoxib in the treatment of symptomatic knee osteoarthritis: a randomized, double-blind, controlled equivalence drug trial. Rheumatology (Oxford). 2013; 52(8):1408-1417.

https://doi.org/10.1093/rheumatology/kes414

10. Cochrane Complementary Medicine. Operational Definition of Complimentary Medicine. https://cam.cochrane.org/operational-definition-complementary-medicine Accessed February 09, 2022.

11. Moher D, Liberati A, Tetzlaff J, Altman DG, Group P. Preferred reporting items for systematic reviews and meta-analyses: the PRISMA statement. PLoS Med. 2009; 6(7):e1000097. https://doi.org/10.1371/journal.pmed.1000097

12. Cohen JF, Deeks JJ, Hooft L, Salameh JP, Korevaar DA, Gatsonis C, Hopewell S, Hunt HA, Hyde CJ, Leeflang MM et al. Preferred reporting items for journal and conference abstracts of systematic reviews and meta-analyses of diagnostic test accuracy studies (PRISMA-DTA for Abstracts): checklist, explanation, and elaboration. BMJ. 2021; 372:n265. https://doi.org/10.1136/bmj.n265 
13. Tricco AC, Lillie E, Zarin W, O'Brien KK, Colquhoun H, Levac D, Moher D, Peters MDJ, Horsley T, Weeks $\mathrm{L}$ et al. PRISMA Extension for Scoping Reviews (PRISMA-ScR): Checklist and Explanation. Ann Intern Med. 2018; 169(7):467-473. https://doi.org/10.7326/M18-0850

14. Arksey H, O'Malley L. Scoping studies: towards a methodological framework. International Journal of Social Research Methodology. 2005; 8(1):19-32. https://doi.org/10.1080/1364557032000119616

15. Levac D, Colquhoun H, O'Brien KK. Scoping studies: advancing the methodology. Implementation Science. 2010; 5(1):69. https://doi.org/10.1186/1748-5908-5-69

16. Colquhoun HL, Levac D, O’Brien KK, Straus S, Tricco AC, Perrier L, Kastner M, Moher D. Scoping reviews: time for clarity in definition, methods, and reporting. J Clin Epidemiol. 2014; 67(12):12911294. https://doi.org/10.1016/j.jclinepi.2014.03.013

17. Otlet P: Traité de documentation: le livre sur le livre, theéorie et pratique. Bruxelles: Editiones Mundaneum; 1934.

18. Rousseau R. Library science: Forgotten founder of bibliometrics. Nature. 2014; $510(7504): 218$. https://doi.org/10.1038/510218e

19. Price DDS. A general theory of bibliometric and other cumulative advantage processes. Journal of the American Society for Information Science. 1976; 27(5):292-306.

https://doi.org/https://doi.org/10.1002/asi.4630270505

20. Hicks D, Wouters P, Waltman L, de Rijcke S, Rafols I. Bibliometrics: The Leiden Manifesto for research metrics. Nature. 2015; 520(7548):429-431. https://doi.org/10.1038/520429a

21. Pritchard A. Statistical Bibliography or Bibliometrics? Journal of Documentation. 1969; 25(4): 348349.

22. Veziari Y, Leach MJ, Kumar S. Barriers to the conduct and application of research in complementary and alternative medicine: a systematic review. BMC Complement Altern Med. 2017; 17(1):166. https://doi.org/10.1186/s12906-017-1660-0

23. Fu J-Y, Zhang $X$, Zhao $Y-H$, Huang $M-H$, Chen D-Z. Bibliometric analysis of complementary and alternative medicine research over three decades. Scientometrics. 2011; 88(2):617-626. https://doi.org/10.1007/s11192-011-0391-0

24. Moral-Munoz JA, Carballo-Costa L, Herrera-Viedma E, Cobo MJ. Production Trends, Collaboration, and Main Topics of the Integrative and Complementary Oncology Research Area: A Bibliometric Analysis. Integrative cancer therapies. 2019; 18:1534735419846401-1534735419846401. https://doi.org/10.1177/1534735419846401

25. Zyoud I, Al-Jabi SW, Sweileh WM. Scientific publications from Arab world in leading journals of Integrative and Complementary Medicine: a bibliometric analysis. BMC Complementary and Alternative Medicine. 2015; 15(1):308. https://doi.org/10.1186/s12906-015-0840-z

26. Vickers AJ. Bibliometric analysis of randomized trials in complementary medicine. Complementary Therapies in Medicine. 1998; 6(4):185-189. https://doi.org/https://doi.org/10.1016/S09652299(98)80026-5 
27. Wieland LS, Manheimer E, Sampson M, Barnabas JP, Bouter LM, Cho K, Lee MS, Li X, Liu J, Moher D et al. Bibliometric and content analysis of the Cochrane Complementary Medicine Field specialized register of controlled trials. Systematic Reviews. 2013; 2(1):51. https://doi.org/10.1186/2046-4053-251

28. Ma Y, Dong M, Zhou K, Mita C, Liu J, Wayne PM. Publication Trends in Acupuncture Research: A 20Year Bibliometric Analysis Based on PubMed. PloS one. 2016; 11(12):e0168123-e0168123. https://doi.org/10.1371/journal.pone.0168123

29. Liang YD, Li Y, Zhao J, Wang XY, Zhu HZ, Chen XH. Study of acupuncture for low back pain in recent 20 years: a bibliometric analysis via CiteSpace. J Pain Res. 2017; 10:951-964. https://doi.org/10.2147/JPR.S132808

30. Şenel E, Demir E. Bibliometric analysis of apitherapy in complementary medicine literature between 1980 and 2016. Complementary Therapies in Clinical Practice. 2018; 31:47-52. https://doi.org/https://doi.org/10.1016/j.ctcp.2018.02.003

31. Jeter PE, Slutsky J, Singh N, Khalsa SB. Yoga as a Therapeutic Intervention: A Bibliometric Analysis of Published Research Studies from 1967 to 2013. J Altern Complement Med. 2015; 21(10):586592. https://doi.org/10.1089/acm.2015.0057

32. Cramer H, Lauche R, Dobos $\mathrm{G}$. Characteristics of randomized controlled trials of yoga: a bibliometric analysis. BMC Complementary and Alternative Medicine. 2014; 14(1):328.

https://doi.org/10.1186/1472-6882-14-328

33. Chiu W-T, Ho Y-S. Bibliometric analysis of homeopathy research during the period of 1991 to 2003. Scientometrics. 2005; 63(1):3-23. https://doi.org/10.1007/s11192-005-0201-7

34. Linnenluecke MK, Marrone M, Singh AK. Conducting systematic literature reviews and bibliometric analyses. Australian Journal of Management. 2019; 45(2):175-194. https://doi.org/10.1177/0312896219877678

35. Bramer WM, de Jonge GB, Rethlefsen ML, Mast F, Kleijnen J. A systematic approach to searching: an efficient and complete method to develop literature searches. J Med Libr Assoc. 2018; 106(4):531541. https://doi.org/10.5195/jmla.2018.283

36. Kugley S, Wade A, Thomas J, Mahood Q, Jørgensen A-MK, Hammerstrøm K, Sathe N. Searching for studies: a guide to information retrieval for Campbell systematic reviews. Campbell Systematic Reviews. 2017; 13(1):1-73. https://doi.org/10.4073/cmg.2016.1

37. Lefebvre C GJ, Briscoe S, Littlewood A, Marshall C, Metzendorf M-I, Noel-Storr A, Rader T, Shokraneh F, Thomas J, Wieland LS. Chapter 4: Searching for and selecting studies Cochrane; 2021. https://www.training.cochrane.org/handbook Accessed February 9, 2022.

38. Salvador-Olivan JA, Marco-Cuenca G, Arquero-Aviles R. Errors in search strategies used in systematic reviews and their effects on information retrieval. J Med Libr Assoc. 2019; 107(2):210-221. https://doi.org/10.5195/jmla.2019.567

39. Wieland LS, Manheimer E, Berman BM. Development and classification of an operational definition of complementary and alternative medicine for the Cochrane collaboration. Altern Ther Health Med. 
2011; 17(2):50-59. https://www.ncbi.nlm.nih.gov/pmc/articles/PMC3196853/

40. Obadia L. The Economies of Health in Western Buddhism: A Case Study of a Tibetan Buddhist Group in France. In: Wood DC. The Economics of Health and Wellness: Anthropological Perspectives. Volume 26. Emerald Group Publishing Limited; 2007. p. 227-259.

41. Braun LA, Tiralongo E, Wilkinson JM, Spitzer O, Bailey M, Poole S, Dooley M. Perceptions, use and attitudes of pharmacy customers on complementary medicines and pharmacy practice. BMC Complementary and Alternative Medicine. 2010; 10(1):38. https://doi.org/10.1186/1472-6882-10-38

42. Shekelle PG, Morton SC, Suttorp MJ, Buscemi N, Friesen C. Challenges in Systematic Reviews of Complementary and Alternative Medicine Topics. Annals of Internal Medicine. 2005; 142(12_Part_2):1042-1047. https://doi.org/10.7326/0003-4819-142-12_Part_2-200506211-00003

43. Vickers A, Goyal N, Harland R, Rees R. Do Certain Countries Produce Only Positive Results? A Systematic Review of Controlled Trials. Controlled Clinical Trials. 1998; 19(2):159-166. https://doi.org/10.1016/S0197-2456(97)00150-5

44. Pham B, Klassen TP, Lawson ML, Moher D. Language of publication restrictions in systematic reviews gave different results depending on whether the intervention was conventional or complementary. J Clin Epidemiol. 2005; 58(8):769-776. https://doi.org/10.1016/j.jclinepi.2004.08.021

45. Wu XY, Tang JL, Mao C, Yuan JQ, Qin Y, Chung VC. Systematic reviews and meta-analyses of traditional Chinese medicine must search Chinese databases to reduce language bias. Evid Based Complement Alternat Med. 2013; 2013:812179. https://doi.org/10.1155/2013/812179

46. Ng JY. Insight into the characteristics of research published in traditional, complementary, alternative, and integrative medicine journals: a bibliometric analysis. BMC Complement Med Ther. 2021 21(185):1-31. https://doi.org/10.1186/s12906-021-03354-7

47. Ng JY, Dhawan T, Dogadova E, Taghi-Zada Z, Vacca A, Wieland S, Moher, D. Operational Definition of Complementary, Alternative, and Integrative Medicine Derived from a Systematic Search. Research Square. 2021. 1-12. https://doi.org/10.21203/rs.3.rs-737412/v1

\section{Supplementary Files}

This is a list of supplementary files associated with this preprint. Click to download.

- SupplementaryFile1SearchStrategyFeb2422.docx

- SupplementaryFile2RemovedTermsFeb2422.docx 